\title{
Performance Analysis and Optimization of Nonhydrostatic ICosahedral Atmospheric Model (NICAM) on the K Computer and TSUBAME2.5
}

\author{
Hisashi Yashiro \\ h.yashiro@riken.jp \\ Shin-ichi Iga \\ iga@riken.jp
}

\author{
Masaaki Terai \\ teraim@riken.jp \\ Kazuo Minami \\ minami_kaz@riken.jp
}

\author{
Ryuji Yoshida \\ ryoshida@riken.jp \\ Hirofumi Tomita \\ htomita@riken.jp
}

RIKEN Advanced Institute for Computational Science

Kobe, Japan 650-0047

\begin{abstract}
We summarize the optimization and performance evaluation of the Nonhydrostatic ICosahedral Atmospheric Model (NICAM) on two different types of supercomputers: the K computer and TSUBAME2.5. First, we evaluated and improved several kernels extracted from the model code on the $\mathrm{K}$ computer. We did not significantly change the loop and data ordering for sufficient usage of the features of the $\mathrm{K}$ computer, such as the hardware-aided thread barrier mechanism and the relatively high bandwidth of the memory, i.e., a 0.5 Byte/FLOP ratio. Loop optimizations and code cleaning for a reduction in memory transfer contributed to a speed-up of the model execution time. The sustained performance ratio of the main loop of the NICAM reached 0.87 PFLOPS with 81,920 nodes on the K computer. For GPUbased calculations, we applied OpenACC to the dynamical core of NICAM. The performance and scalability were evaluated using the TSUBAME2.5 supercomputer. We achieved good performance results, which showed efficient use of the memory throughput performance of the GPU as well as good weak scalability. A dry dynamical core experiment was carried out using 2560 GPUs, which achieved 60 TFLOPS of sustained performance.
\end{abstract}

\section{CCS Concepts}

- Applied computing $\rightarrow$ Physical sciences and engineering; Earth and atmospheric sciences;

\section{Keywords}

Weather; Climate; GCM; K Computer; TSUBAME2.5; OpenACC; Extreme-Scale Computing; Memory-Bound

\section{INTRODUCTION}

Permission to make digital or hard copies of all or part of this work for personal or classroom use is granted without fee provided that copies are not made or distributed for profit or commercial advantage and that copies bear this notice and the full citation on the first page. Copyrights for components of this work owned by others than the author(s) must be honored. Abstracting with credit is permitted. To copy otherwise, or republish, to post on servers or to redistribute to lists, requires prior specific permission and/or a fee. Request permissions from permissions@acm.org.

PASC '16, June 08-10, 2016, Lausanne, Switzerland

(C) 2016 Copyright held by the owner/author(s). Publication rights licensed to ACM. ISBN 978-1-4503-4126-4/16/06 . .\$15.00

DOI: http://dx.doi.org/10.1145/2929908.2929911
The Nonhydrostatic ICosahedral Atmospheric Model (NICAM) $[21,20,30]$ has been continuously developed since 2000. A major motivation for developing the NICAM was the challenge of simulating a tropical cloud system in the global domain without any convection parameterization. Deep convective systems in the tropics play important roles in global atmospheric circulations. Each deep convective core has a horizontal scale of $\mathrm{O}(\mathrm{km})$. However, the horizontal resolution of conventional global climate models was $\mathrm{O}(100 \mathrm{~km})$ in the $2000 \mathrm{~s}$. Work has begun to design the NICAM for global simulation with a 3.5$\mathrm{km}$ horizontal mesh by using the Earth Simulator (http: //www.jamstec.go.jp/es/en/), which was launched by the Japan Agency for Marine-Earth Science and Technology (JAMSTEC) in 2002. Firstly, an aqua-planet experiment was performed at this horizontal resolution[29]. The results showed that the cloud-system resolving simulation is promising for the expression of a multiscale convective structure and the diurnal cycle of precipitation in the tropics. With the introduction of real topography and land-sea contrast, Miura et al.[13] succeeded in simulating a realistic MaddenJulian oscillation with several kilometers of horizontal mesh without any cumulus parameterization. Following these simulations, many studies have been conducted by using the NICAM (see the detailed scientific overview in [20]).

The K computer - the first Japanese ten-petaflops supercomputer - was launched in 2011 and has been providing large computational resources to the climate research area. We extended the simulation with a $14-\mathrm{km}$ horizontal mesh to 30 years[11]. Further, the first global subkilometer simulation has been conducted[14]. The results showed that the deep convective core tends to be resolved by multiple grids with an 870-m horizontal mesh. These studies are supported by the efforts of optimization on the $\mathrm{K}$ computer, which is described in this paper.

On the basis of scientific interest, we need to execute larger-scale simulations with regard to several aspects such as the spatial resolution, the duration time, the ensemble size, and the usage of more sophisticated submodules. A state-of-the-art supercomputer, which will realize these simulations, will desirably have high-performance memory throughput and power efficiency, which may be possibly realized by GPU-based supercomputers. Shimokawabe 
et al.[23] ported and optimized the mesoscale atmosphere model ASUCA $[3,8]$ on TSUBAME2.0 and achieved good performance. The code was rewritten on CUDA for GPU calculation, and a new $\mathrm{C}++$-based framework was then introduced into it[24]. In recent years, a new directive-based programming model called OpenACC has enabled the effective and easy use of GPUs. OpenACC is used to port part of the regional weather and climate model COSMO[4], which has already been used for operational weather forecasting on GPU-based supercomputers.

In this paper, we detail the optimization on two different types of Japanese supercomputers: the K computer and TSUBAME2.5. In Section 2, we summarize the characteristics of the NICAM code. The history of optimization and the performance results are described in Section 3. We detail the GPU implementation and present a performance comparison of TSUBAME2.5 and the K computer in Section 4. We summarize and discuss the results and future plans in Section 5 .

\section{THE DESIGN AND SOURCE CODE OF NICAM}

Currently, the NICAM continues to be developed mainly by JAMSTEC, the University of Tokyo, and the RIKEN Advanced Institute of Computational Science (AICS). Since the detailed development history of the NICAM is described by Satoh et al.[21], only a short summary is presented here. A three-dimensional dynamical core was developed by combining two approaches; one is the construction of a shallowwater model on the icosahedral grid system[31, 32]. To improve the numerical accuracy and stability, a modification of the grid points with the spring dynamics method was proposed and evaluated. The other approach is the development of new nonhydrostatic schemes[18, 19]. These nonhydrostatic schemes are based on the flux form equations, and the conservation of the mass and total energy was taken into consideration. For a larger step interval $(\Delta t)$, the split-explicit time integration scheme[10] is used with the Horizontally Explicit and Vertically Implicit (HE-VI) scheme. Then, full-physics experiments were started in 2004. Cloud microphysics schemes[5, 22, 27] and boundary-layer schemes[15, 16, 17] are actively developed and/or have been implemented into the model. In recent years, the Center for Climate System Research (CCSR) Ocean Component Model (COCO) $[6,7]$ has been coupled with the NICAM by using the Jcup library $[1,2]$ for atmosphere-ocean coupling simulations. Data assimilation (DA) systems based on the NICAM have been actively developed. Terasaki et al.[26] proposed an ensemble-based DA system for weather prediction by using a Local Ensemble Transform Kalman filter (LETKF). A new workflow, which is focused on data movement in the NICAM-LETKF DA system, has been proposed[33]. The inversion system of trace gases with the four-dimensional variational (4D-Var) method has also been developed and evaluated (Niwa et al., in preparation).

The NICAM has approximately 310,000 lines of code. Most of the code is written in Fortran 90, except the lowlevel I/O module. The source code of the NICAM is roughly divided into four categories: driver+utilities, dynamics, physics, and pre/post-process tools. Driver+utilities consists of the main programs and modules for administration, input/output, variable containers, and general-purpose

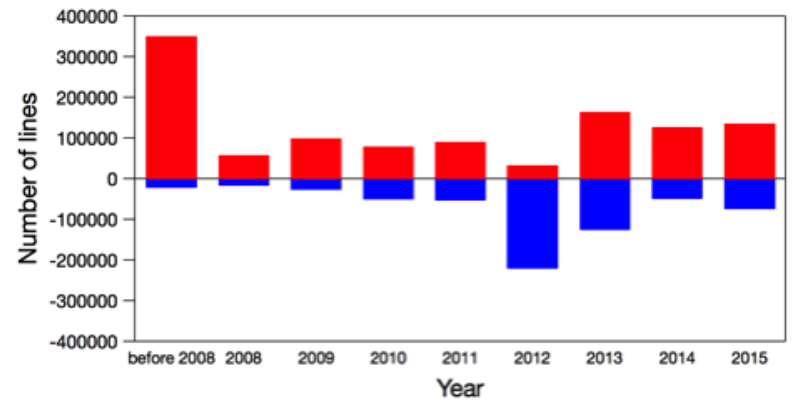

Figure 1: Modified lines of NICAM code. Red and blue bars indicate added and deleted lines, respectively. The data are summarized from the commit log of the version control system.

functions. This part has 40,000 lines of code. Dynamics is the dynamic core part and has 60,000 lines of code. This part contains the node-to-node communication module for halo exchange. The physics part contains the physical schemes and models such as the cloud microphysics, boundary layer, surface flux, and atmospheric radiation. This part takes up the largest portion of the model code and has 130,000 lines. The pre/post-process tools are also important as well as the part for the time integration. The preprocess tools convert from an external dataset such as the topography, land-use category, and reanalysis data to model the initial data or boundary data. Post-process tools are used for regridding from icosahedral grids to latitude-longitude grids horizontally, conversion from height coordinates to pressure coordinates vertically, calculating some diagnostic values, and so on. This part has 80,000 lines of code.

Figure 1 shows the lines added to and deleted from the NICAM code, which are summarized from the commit log of the version control system. During last 8 years, tens of thousands of lines have been added and/or deleted every year. The development of physics schemes and post-process tools is most active. During 2011 and 2013, every part was modified because of the porting projects on the $\mathrm{K}$ computer. Such active replacement of the code lines is often done for the development of the weather/climate model. Sometimes, this makes it difficult to maintain a high computing speed owing to the modification of optimized code and the incorporation of new naive code.

The NICAM was developed for calculation on a parallel vector machine at first. For good vectorization and parallelization efficiency, we treated the icosahedral grid as a kind of structured grid. We also applied the recursive division method to both the grid points and grid groups for each process[28]. Figure 2 shows an example of the grid and grid group division. The number of horizontal grids on the sphere increases from Figures 2(a)-2(c) with the increasing division level of the grid. If we divide nine times, the total number of horizontal grids reaches 2,621,442, which corresponds to a spacing on the Earth's sphere of $\sim 14 \mathrm{~km}$. Figure 2(d) shows the minimum division level of the computational region. Every two neighboring triangles of the icosahedron are combined into a diamond. This diamond is called a "region." The same number of grids is included in each region. Each region also has a halo for exchanging data from neighboring regions. The ten diamonds in Figure 2(d) 
(a)

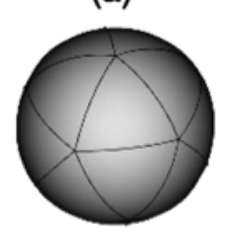

(b)

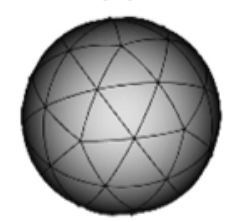

Grid Division

(d)

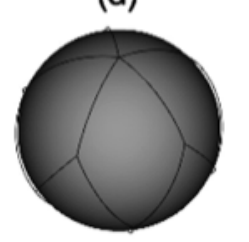

(e)
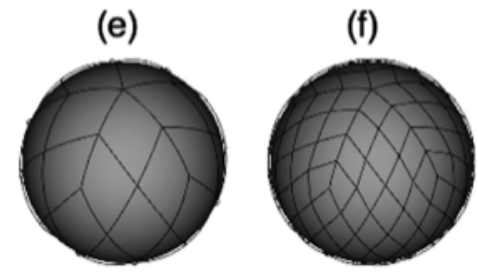

Computational Region Division

(c)

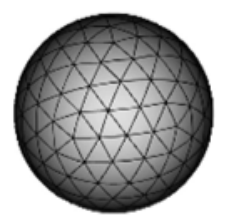

\section{.}

table feature of the $\mathrm{K}$ computer is the hardware barrier synchronization among the cores within a CPU. This mechanism can synchronize multiple threads approximately ten times faster than software barrier processing. Thus, we can reduce the overhead at the level of fine-grained parallelism and obtain very good scalability of parallel threads[12].

As described by Terai et al[25], six kernels were extracted from the dynamics and two from the physics in the NICAM code. The $\mathrm{K}$ computer prepares two ways of thread parallelization: automatic parallelization and OpenMP directives. At the time of porting the NICAM code to the $\mathrm{K}$ computer, there were no OpenMP directives in the code. We found that the kernels compiled by automatic parallelization showed good performance as well as the kernels applying OpenMP. Thus, we decided to use automatic parallelization. The advantage of automatic parallelization is that it is not necessary to write any OpenMP directives. Indeed, we did not make any efforts for debugging with OpenMP. However, OpenMP is necessary if we want to consider a large parallel region. We have a plan to use OpenMP in the future.

We attempted various optimizations such as array merging, array dimension interchange, loop fission, loop unrolling, and compiler option optimization. In general, we did not change the data ordering between the horizontal and vertical dimensions, for example, changing from $(i j, k)$ to $(k, i j)$. We assigned priority to a long and continuous memory access of the data array rather than the cache blocking. Thread parallelization was applied to the $k$ loop, and software pipelining and SIMD execution were applied to $i j$ loop. The refactored and optimized kernels showed good performance. For example, the performance efficiency of the stencil kernel for the divergence operator improved from $3.5 \%$ to $16.8 \%$. We applied these kernels to the application and evaluated the total performance.

For the benchmark test, we used a realistic case of global atmosphere simulation. Boundary conditions were prepared for different horizontal resolutions. The initial conditions were obtained from the linearly interpolated data from the analytical dataset. The simulation was executed with all of the key physics components. The main loop was executed at least 10 times without any file output during the benchmark. Just after porting to the $\mathrm{K}$ computer, the peak performance of the main loop was approximately $4 \%$ for the single-node performance of the benchmark test. After the optimization of the stencil kernel of the dynamics, we only obtained $\sim 5 \%$ of the total performance. This result was related to the effect of Ahmdahl's law. Stencil kernels were frequently called in the dynamics, but their execution time became only a small portion of the total time. To find the new bottleneck, we inserted several hundreds of floating operation counters and timers into the critical path of the source code. The analysis results of this labor-intensive method resulted in much knowledge to our community, particularly:

- There were many occurrences of unnecessary zero filling for a large array for initialization.

- There were too many intermediate arrays. Large arrays were used for the calculation with a very low arithmetic intensity.

- Conditional branches were inserted into the innermost loops. This was because the person who updated a scheme based on a new study wanted to maintain backwards compatibility. 

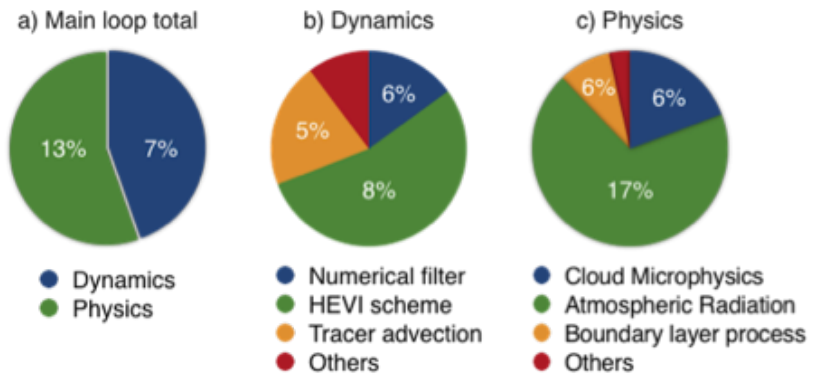

Figure 3: A breakdown of the contribution of each component to the elapsed time for the a) main loop, b) dynamics, and c) physics. The percentages indicate the peak performance of each component.

These time-consuming parts in the code are difficult to find. When we used some profilers with the sampling analysis, we were able to find the most time-consuming part. However, each part listed above was small and distributed anywhere in the code. We could not find them in the cost ranking of the profiler. According to the information from the detailed counter and timers, we removed the time-consuming part. After minor optimization work, the performance efficiency finally reached $10.2 \%$. We also succeeded in reducing the model execution time by $30 \%$.

Figure 3 shows a summary of the single-node performance of the NICAM on the K computer. The execution times of the dynamics and physics (indicated by the pie areas in Figure 3(a)) are almost equal in the main loop. The computational efficiency (indicated by the percentages) of the dynamics is relatively low owing to the low arithmetic intensity and the network communication for halo exchange. The communication kernel is frequently called in the numerical filter scheme to apply high-order Laplacian filtering. The tracer advection scheme indicates another issue. The max and min functions and conditional branches are used for correction of the tracer advection flux in this scheme. These two parts are the major performance bottlenecks for the dynamics (see Figure 3(b)). For the physics, three components mainly consume the execution time (Figure $3(\mathrm{c})$ ). The atmospheric radiation takes up a large portion of the execution time and shows high sustained performance. The cloud microphysics part includes a 1-dimensional advection scheme with a flux limiter for the gravitational falling of rain, snow, and graupel, which has a lower computational efficiency.

We evaluated the weak scalability by changing the number of nodes from 5 to 81,920 nodes. To maintain the same problem size per process, the horizontal grid spacing was decreased as the number of nodes increased. Figure 4 shows the elapsed time and sustained FLOPS in the weak scaling test. The results show good scalability from 5 to 81,920 nodes while maintaining $8.3-10.2 \%$ of performance efficiency. We found that the increase in the elapsed time was mainly due to the load imbalance in the cloud microphysics scheme. The number of iterative calculations, which is called the "saturation adjustment," is highly dependent on the distribution of water vapor and clouds worldwide. Since we adopted horizontal decomposition for MPI parallelization with same number of grids, this kind of load imbalance related to the inhomogeneous distribution of the atmospheric

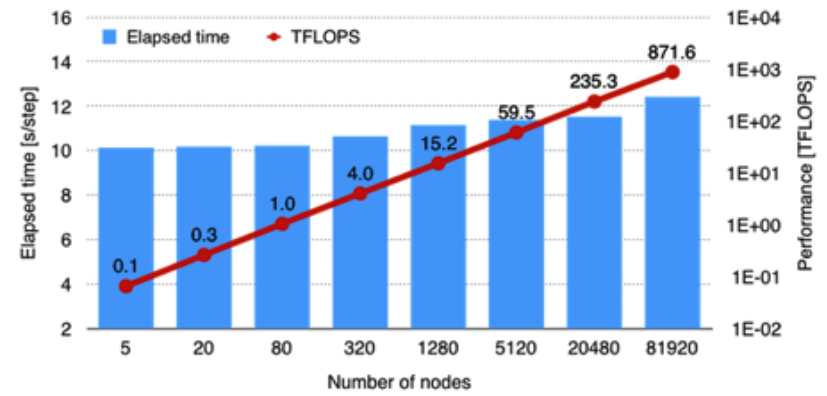

Figure 4: The results of the weak scaling test on the $K$ computer. Blue bars show the elapsed time, and the red line indicates the sustained performance.

composition will become larger in future high-resolution atmospheric simulations with a larger number of processes.

\section{PERFORMANCE OPTIMIZATION AND EVALUATION ON TSUBAME2.5}

The TSUBAME 2.5 supercomputer at the Tokyo Institute of Technology has 1,048 compute nodes, each of which is equipped with three NVIDIA Tesla K20X GPUs. Each node has two Intel Xeon X5670 (Westmere) hexacore CPUs and two QDR InfiniBand interconnects for connection to the fullbisection dual-rail fat-tree network. The peak performance of the system in double precision is 5.76 PFLOPS.

We started to optimize the NICAM code without the physics part. Optimization of the physics part is an ongoing project at the time of writing this paper. Several parts of the drivers, utilities, and dynamics were repackaged as NICAM-DC. NICAM-DC is distributed under the BSD 2-clause license (http://scale.acis.riken.jp/nicamdc/). Further, NICAM-DC is a standalone application and includes some popular idealized test cases for the dynamical core of the global atmospheric model. With the following strategies, NICAM-DC was optimized for GPUs using OpenACC:

- Memory allocation for all of the arrays is conducted during the setup stage before the main loop. At the end of the setup stage, the precalculated coefficients and metrics are transferred to the device using "data pcopyin." These arrays are not changed and are kept in the GPU memory during calculation of the main loop.

- The "async" clause is used to loop kernels as much as possible. If we use this feature, the loop kernels are asynchronously executed on the GPU while the host process continues the next part of the code. For this purpose, the timing of the halo exchange communications is rearranged in some parts.

- Parallel execution is carried out on the CPU and GPU. In the NICAM code, some small computations are at the side of the large stencil loop kernel. They are executed on the host process. This is one advantage of OpenACC for readability of the code. If we use the CUDA programming model, we have to separate the offloading loops and nonoffloading loops to different kernel functions. OpenACC enables more flexible 
a) TGPU

b) TCPU

c) KCPU
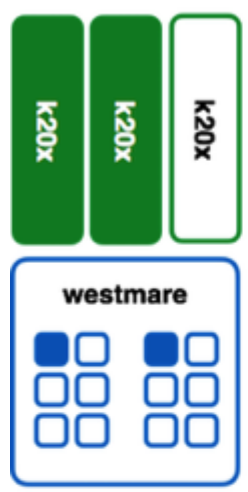

TSUBAME2.5

GPU-CPU

$2 \mathrm{MPI} /$ node

$1 \mathrm{GPU} / \mathrm{MPI}$

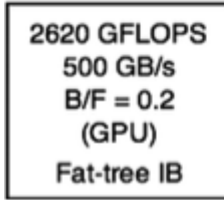

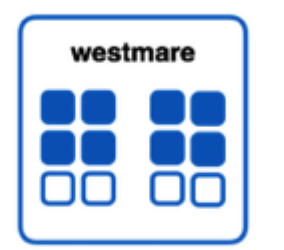

TSUBAME2.5

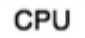

$8 \mathrm{MPI} /$ node flat MPI

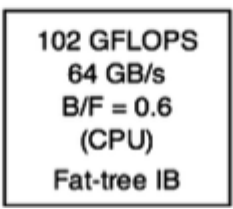

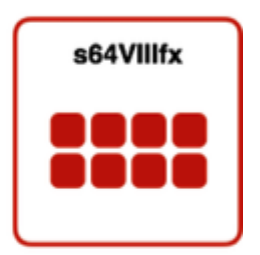

the $\mathrm{K}$ computer CPU

$1 \mathrm{MPI} /$ node 8 threads / MPI

\begin{tabular}{|c|}
\hline $128 \mathrm{GFLOPS}$ \\
$64 \mathrm{~GB} / \mathrm{s}$ \\
$\mathrm{B} / \mathrm{F}=0.5$ \\
(CPU) \\
Tofu \\
\hline
\end{tabular}

Figure 5: Configuration of the usage of CPUs and GPUs in the nodes of TSUBAME2.5 and the $\mathrm{K}$ computer.

treatment regarding whether each loop uses the GPU within a subroutine.

- Data packing/unpacking for halo communication is conducted on the GPU. The number of grids required for halo exchange is smaller than the total number of grids. By processing data packing on GPU, we can minimize the data transfer between the device and the host.

- Frequent file output is treated in the main loop. We calculate the diagnosed variables to output and convert their vertical coordinates on the GPU. At the time of output, which is usually every tens or hundreds of main-loop steps, these data are transferred from the device.

As described above, we did not change the data ordering since we mainly used a vector machine. The size of the inner loop of the loop nests is the largest in most cases. Recurrence of the data is sometimes found in the outer loop, i.e., the $k$ loops. These data and loop structure are fitted to the GPU computing. Approximately 2,000 lines of code were added and deleted for GPU optimization. The major modification was the insertion of OpenACC directives.

We executed a benchmark simulation of NICAM-DC to evaluate the performance on the TSUBAME2.5 supercomputer. The same experiments were conducted on the K computer for comparison. The benchmark test was based on a baroclinic instability test case[9], and only small steps were executed. The total number of grid points of the control run was 26 million with a $56-\mathrm{km}$ horizontal mesh and 160 vertical layers. We included the frequent file output of the

TGPU (TSUBAME2.5 $2 \mathrm{MPI}$ processes per node + 2GPUs) TCPU (TSUBAME2.5 8 MPI processes per node)

KCPU (The K computer $1 \mathrm{MPI}$ process per node $\times 8$ threads)
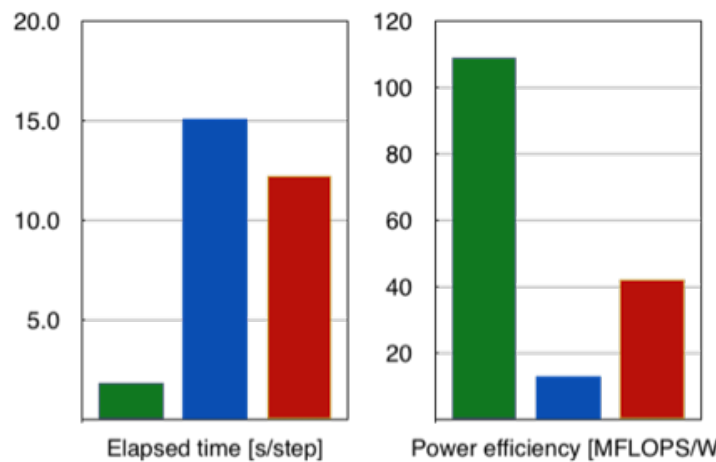

Power efficiency [MFLOPS/W]

Figure 6: Performance results of NICAM-DC on TSUBAME2.5 and the $K$ computer for (left) the elapsed time for the benchmark test and (right) the power efficiency.

variables for analysis in this benchmark. Three different configurations of the architecture were used for a node-to-node comparison, as shown in Figure 5. The first is for CPUGPU calculation on TSUBAME2.5 (TGPU, Figure 5(a)). We only used two GPUs of each node of TSUBAME2.5 owing to the limitations of the process division of the NICAM. The second is CPU-only calculation using TSUBAME2.5 (TCPU, Figure 5(b)). In this case, the flat MPI approach was used, and the number of MPI processes increased fourfold in comparison to the CPU-GPU case. The last is for CPU-only calculation with the hybrid thread-MPI approach on the K computer (KCPU, Figure 5(c)). To keep almost the same problem size for each node of three configurations, the problem size for each process was changed by using the division method of the NICAM. The size ratios of the problems among the three configurations were adjusted to 1:0.25:2. For the TGPU configuration, the total number of floatingpoint operations in the main loop was 420 GFLOP, and 2.3 TB/step of memory transfer was required for each MPI process in the node. We used monitoring tools for the power consumption of each node and each GPU in TSUBAME2.5. The power consumption rate was also measured on the $\mathrm{K}$ computer in a similar manner.

Figure 6 shows the results for single-node performance. The left panel shows a comparison of the elapsed time. The TGPU configuration is approximately eight and seven times faster than TCPU and KCPU, respectively. This result is approximately proportional to the difference in the memory performance. The difference in performance between TCPU and KCPU is mainly caused by the difference in the approach to parallelization - flat MPI and hybrid. We achieved a peak memory transfer of $\sim 50 \%$ for the GPU calculation. This result shows that the code generated by OpenACC demonstrates good performance as well as the code written by CUDA. The right panel in Figure 6 shows a comparison of the power consumption in MFLOPS per watt. The difference between the TGPU and TCPU configurations is approximately inversely proportional to the elapsed time. The result on the $\mathrm{K}$ computer (KCPU) shows better power efficiency compared to TCPU. In addition, the calculation using the GPU was the most efficient in terms of 


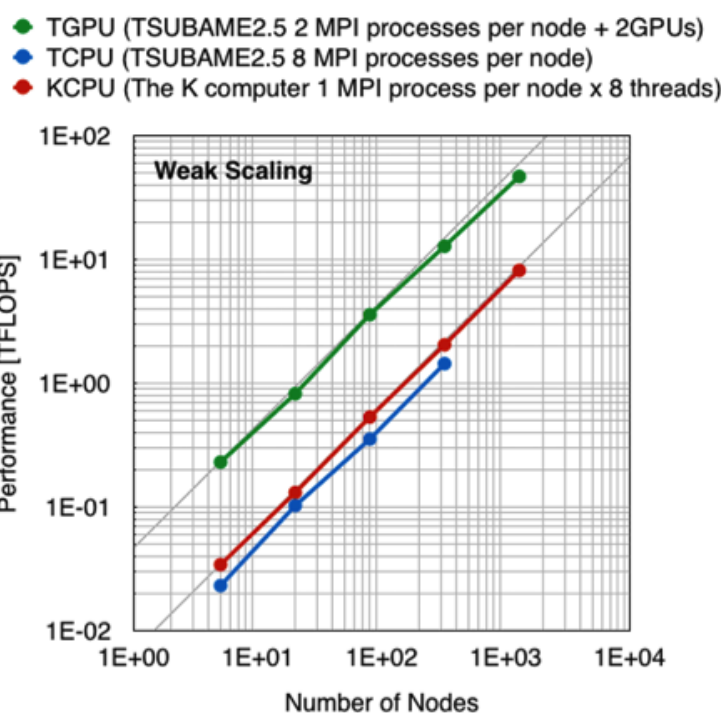

Figure 7: Weak scaling results of NICAM-DC on TSUBAME2.5 and the $K$ computer.

electricity consumption among the three configurations.

Weak scaling tests were performed by increasing from 5 nodes to 1,280 nodes (Figure 7). The horizontal mesh size was decreased from $56 \mathrm{~km}$ to $3.5 \mathrm{~km}$, and the total number of grids was increased according to the number of nodes used. The results show that all three configurations show good scalability. The largest simulation for TGPU used 2,560 GPUs and achieved 46.5 TFLOPS. The main cause of the increase in the elapsed time was file I/O in the CPUGPU calculation. The ratio of the elapse time of file I/O was around $2 \%$ in the every test of TCPU and KCPU configuration. However, the TGPU configuration showed large percentages of $\mathrm{I} / \mathrm{O}$ time. The ratio increased from $13 \%$ to $28 \%$ as the nodes size is increased from 5 to 1,280 . We tested longer intervals for data output, which is usual for a productive run (every $12 \mathrm{~h}$ in simulation time). In this case, the performance reached 60 TFLOPS by using 1,280 nodes (2,560 GPUs).

Figure 8 shows the results of strong scaling tests. The number of nodes increased from 5 to 1,280 without changing the horizontal resolution. The problem size was same as the performance benchmark test. From the results, we found rapid performance saturation for the TGPU configuration when we increased the number of nodes. This was related to the decrease in the horizontal grid size per process. As the number of nodes is increased from 5 to 1,280 , the number of horizontal grids (including the halo grid) in each process was changed from 33,800 to 200 . This change results in a decrease in the size of the innermost loop. A loop size that is too small is not sufficient for the execution of threads on the GPU. A similar but slower deterioration in the scalability is found for the KCPU configuration. Moreover, there is not enough work for efficient calculation with the OpenMP-type thread parallelization in this case. We used compiler-aided thread parallelization on the K computer, which uses smaller parallel region for threading. We may get better results for strong scaling by using OpenMP and optimizing for this problem size. The ratio of the communication time to the time for computation increased when we used a large number
- TGPU (TSUBAME2.5 2 MPI processes per node + 2GPUs)

- TCPU (TSUBAME2.5 8 MPI processes per node)

- KCPU (The K computer 1 MPI process per node $\times 8$ threads)

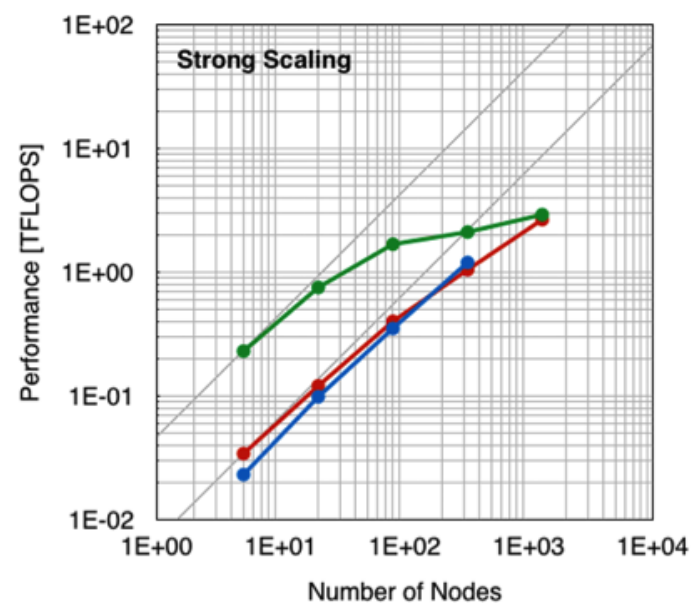

Figure 8: Strong scaling results of NICAM-DC on TSUBAME2.5 and the $\mathrm{K}$ computer.

of nodes. To decrease the communication time, we should reduce the number of occurrences of MPI communication. The performance of strong scaling is more critical for climate simulations, which integrates $10^{6}-10^{7}$ steps. In the future, more improvements are required for the purpose of this type of simulation.

\section{SUMMARY AND FUTURE PLANS}

In this paper, we have presented an overview of the performance results of a global high-resolution atmospheric model on two supercomputers. We succeeded in maintaining the portability of the NICAM code with good performance and scalability. Now, simulations using the NICAM can be executed on many types of supercomputers such as parallel vector machines, massively parallel scalar machines, and GPU-based machines. Many optimization efforts brought about knowledge of the importance of data movement everywhere in the code. For the continuous integration of new components and schemes into our model, we should keep reducing the data movement. In particular, many variables are shared among the plural modules in the physics part. These modules are developed at different times and by different developers. To avoid making unnecessary arrays, it is important to continue refactoring among these modules.

Weather and climate models are large, complex, dataintensive applications in high-performance computing. For more efficient calculation, we need a higher throughput for data transfer among many components in the supercomputer system, such as the memory, GPU-CPU connections, internode communication, and file I/O. Data movement is highly connected to the power consumption of the computer system. Our model development will have to take care of the data movement. The active use of mixed precision is one possible solution. We may reduce the redundant use of double precision by evaluating the simulation results at the same time as the code optimization using mixed precision. File I/O is also critical issue. In GPU-based simulations, a large amount of data is transferred from the GPU to the 
CPU when we want to output results during the simulation. To reduce such a large transfer of data, compression will be effective. The libraries, which compress the data on the GPU with a common format such as NetCDF and HDF, will be useful for the weather and climate modeling communities.

In the future, it will be more difficult to keep both code portability and performance. The use of a domain specific language (DSL) will be promising approach to solve this issue. For example, the STELLA DSL is used for rewriting the dynamical core of the COSMO model[4]. We have a plan to use such DSLs to support future model development and the whole tool ecosystem for weather and climate studies.

\section{ACKNOWLEDGMENTS}

Some of the simulations were performed using the K computer at the RIKEN Advanced Institute for Computational Science. The authors thank the Global Scientific Information and Computing Center, the Tokyo Institute of Technology, for use of the resources of the TSUBAME2.5 supercomputer. The largest simulation with GPUs was executed as a TSUBAME Grand Challenge Program, spring 2014. This research was partly supported by the G8 Research Councils Initiative for Multilateral Research Funding, "Icosahedral-Grid Models for Exascale Earth System Simulations (ICOMEX)."

The authors would like to thank Kiyotaka Sakamoto at Fujitsu Systems East Ltd. for his contribution to the optimization on the $\mathrm{K}$ computer. We would also like to thank Akira Naruse at NVIDIA Corp. for help with the OpenACC implementation.

\section{REFERENCES}

[1] T. Arakawa, T. Inoue, and M. Sato. Performance evaluation and case study of a coupling software ppOpen-MATH/MP. Procedia Comput. Sci., 29:924-935, 2014.

[2] T. Arakawa, H. Yoshimura, F. Saito, and K. Ogochi. Data exchange algorithm and software design of KAKUSHIN coupler Jcup. Procedia Comput. Sci., 4:1516-1525, 2011.

[3] K. Aranami, T. Hara, Y. Ikuta, K. Kawano, K. Matsubayashi, H. Kusabiraki, T. Ito, T. Egawa, K. Yamashita, Y. Ota, Y. Ishikawa, T. Fujita, and J. Ishida. A new operational regional model for convection-permitting numerical weather prediction at JMA. CAS/JSC WGNE Research Activities in Atmospheric and Oceanic Modelling, 2015.

[4] O. Fuhrer, C. Osuna, X. Lapillonne, T. Gysi, B. Cumming, M. Bianco, A. Arteaga, and T. C. Schulthess. Towards a performance portable, architecture agnostic implementation strategy for weather and climate models. Supercomput. Frontiers Innov., 1(1):45-62, 2014.

[5] W. W. Grabowski. Toward cloud resolving modeling of large-scale tropical circulations: A simple cloud microphysics parameterization. J. Atmos. Sci., 55:3283-3298, 1998.

[6] H. Hasumi. CCSR ocean component model (COCO). CCSR Rep 13. The University of Tokyo, Chiba, Japan, 2000.

[7] H. Hasumi. CCSR ocean component model (COCO), version 4.0. CCSR Rep 25. The University of Tokyo,
Chiba, Japan, 2006.

[8] J. Ishida, C. Muroi, and K. Kawano. Development of a new nonhydrostatic model ASUCA at JMA. CAS/JSC WGNE Research Activities in Atmospheric and Oceanic Modelling, 2010.

[9] C. Jablonowski and D. L. Williamson. A baroclinic instability test case for atmospheric model dynamical cores. Q. J. R. Meteorol. Soc., 132(621C):2943-2975, 2007.

[10] J. B. Klemp and R. B. Wilhelmson. The simulation of three-dimensional convective storm dynamics. $J$. Atmos. Sci., 35(6):1070-1096, 1978.

[11] C. Kodama, Y. Yamada, A. T. Noda, K. Kikuchi, Y. Kajikawa, T. Nasuno, T. Tomita, T. Yamamura, H. G. Takahashi, M. Hara, Y. Kawatani, and M. Satoh. A 20-year climatology of a NICAM AMIP-type simulation. J. Meteorol. Soc. Japan, 93(4):393-424, 2015.

[12] Y. Matsuzaki, N. Uchikoga, M. Ohue, T. Shimoda, T. Sato, T. Ishida, and Y. Akiyama. MEGADOCK 3.0: A high-performance protein-protein interaction prediction software using hybrid parallel computing for petascale supercomputing environments. Source Code Biol. Med., 8(1):18, 2013.

[13] H. Miura, M. Satoh, T. Nasuno, A. T. Noda, and K. Oouchi. A Madden-Julian oscillation event realistically simulated by a global cloud-resolving model. Science, 318(5857):1763-1765, 2007.

[14] Y. Miyamoto, Y. Kajikawa, R. Yoshida, T. Yamaura, H. Yashiro, and H. Tomita. Deep moist atmospheric convection in a subkilometer global simulation. Geophys. Res. Lett., 40(18):4922-4926, 2013.

[15] M. Nakanishi and H. Niino. An improved Mellor-Yamada level-3 model: Its numerical stability and application to a regional prediction of advection fog. Bound.-Layer Meteorol., 119(2):397-407, 2006.

[16] M. Nakanishi and H. Niino. Development of an improved turbulence closure model for the atmospheric boundary layer. J. Meteorol. Soc. Japan, 87(5):895-912, 2009

[17] A. T. Noda, K. Oouchi, M. Satoh, H. Tomita, S. Iga, and Y. Tsushima. Importance of the subgrid-scale turbulent moist process: Cloud distribution in global cloud-resolving simulations. Atmos. Res., 96(2-3):208-217, 2010.

[18] M. Satoh. Conservative scheme for the compressible nonhydrostatic models with the horizontally explicit and vertically implicit time integration scheme. Mon. Wea. Rev., 130(5):1227-1245, 2002.

[19] M. Satoh. Conservative scheme for a compressible nonhydrostatic model with moist processes. Mon. Wea. Rev., 131(6):1033-1050, 2003.

[20] M. Satoh, T. Matsuno, H. Tomita, H. Miura, T. Nasuno, and S. Iga. Nonhydrostatic icosahedral atmospheric model (NICAM) for global cloud resolving simulations. J. Comput. Phys., 227(7):3486-3514, 2008.

[21] M. Satoh, H. Tomita, H. Yashito, H. Miura, C. Kodama, T. Seiki, A. T. Noda, Y. Yamada, D. Goto, M. Sawada, T. Miyoshi, Y. Niwa, M. Hara, T. Ohno, S. Iga, T. Arakawa, T. Inoue, and H. Kubokawa. The Non-hydrostatic Icosahedral 
Atmospheric Model: Description and development. Prog. Earth Planet. Sci., 1(1):18, 2014.

[22] T. Seiki and T. Nakajima. Aerosol effects of the condensation process on a convective cloud simulation. J. Atmos. Sci., 71(2):833-853, 2014.

[23] T. Shimokawabe, T. Aoki, J. Ishida, K. Kawano, and C. Muroi. 145 TFlops performance on 3990 GPUs of TSUBAME 2.0 supercomputer for an operational weather prediction. Procedia Comput. Sci., 4:1535-1544, 2011.

[24] T. Shimokawabe, T. Aoki, and N. Onodera. Performance optimization and evaluation of a global climate application using a $440 \mathrm{~m}$ horizontal mesh on the K computer. In SC14: The International Conference for High Performance Computing, Networking, Storage and Analysis, pages 251-261. IEEE, November 2014.

[25] M. Terai, H. Yashiro, K. Sakamoto, S. Iga, and H. Tomita. High-productivity framework on GPU-rich supercomputers for operational weather prediction code ASUCA. In SC14: The International Conference for High Performance Computing, Networking, Storage and Analysis, November 2014.

[26] K. Terasaki, M. Sawada, and T. Miyoshi. Local ensemble transform Kalman filter experiments with the Nonhydrostatic Icosahedral Atmospheric Model NICAM. SOLA, 11:23-26, 2015.
[27] H. Tomita. New microphysical schemes with five and six categories by diagnostic generation of cloud ice. $J$. Meteorol. Soc. Japan, 86A:121-142, 2008.

[28] H. Tomita, K. Goto, and M. Satoh. A new approach to atmospheric general circulation model: Global cloud resolving model NICAM and its computational performance. SIAM J. Sci. Comput., 30(6):2755-2776, 2008.

[29] H. Tomita, H. Miura, S. Iga, T. Nasuno, and M. Satoh. A global cloud-resolving simulation: Preliminary results from an aqua planet experiment. Geophys. Res. Lett., 32(8):L08805, 2005.

[30] H. Tomita and M. Satoh. A new dynamical framework of nonhydrostatic global model using the icosahedral grid. Fluid Dyn. Res., 34(6):357-400, 2004.

[31] H. Tomita, M. Satoh, and K. Goto. An optimization of the icosahedral grid modified by spring dynamics. J. Comput. Phys., 183(1):307-331, 2002.

[32] H. Tomita, M. Tsugawa, M. Satoh, and K. Goto. Shallow water model on a modified icosahedral geodesic grid by using spring dynamics. J. Comput. Phys., 174(2):579-613, 2001.

[33] H. Yashiro, K. Terasaki, T. Miyoshi, and H. Tomita. Performance evaluation of throughput-aware framework for ensemble data assimilation: The case of NICAM-LETKF. Geosci. Model Dev. Discuss., submitted. 